\begin{tabular}{|c|l|}
\hline Title & A pplication of the quantum spin glass theory to image restoration \\
\hline Author(s) & Inoue, Jun-ichi \\
\hline Citation & $\begin{array}{l}\text { Physical Review E, 63(4), (046114)1-(046114)10 } \\
\text { https://doi.org/40.1103/PhysRevE.63.046114 }\end{array}$ \\
\hline Issue Date & 2001-03-29 \\
\hline Doc URL & http://hdl.handle.net/2115/5421 \\
\hline Rights & Copyright $\odot 2001$ American Physical Society. \\
\hline Type & article \\
\hline File Information & PRE63-4.pdf \\
\hline
\end{tabular}

Instructions for use 


\title{
Application of the quantum spin glass theory to image restoration
}

\author{
Jun-ichi Inoue \\ Complex Systems Engineering, Graduate School of Engineering, Hokkaido University, N13-W8, Kita-ku, Sapporo, 060-8628, Japan
}

(Received 27 June 2000; published 29 March 2001)

\begin{abstract}
Quantum fluctuation is introduced into the Markov random-field model for image restoration in the context of a Bayesian approach. We investigate the dependence of the quantum fluctuation on the quality of a black and white image restoration by making use of statistical mechanics. We find that the maximum posterior marginal (MPM) estimate based on the quantum fluctuation gives a fine restoration in comparison with the maximum a posteriori estimate or the thermal fluctuation based MPM estimate.
\end{abstract}

DOI: 10.1103/PhysRevE.63.046114

PACS number(s): 02.50.-r, 05.20.-y, 05.50.+q

\section{INTRODUCTION}

Recently, the problems of information science have been investigated from a statistical mechanical point of view. Image restoration is one of the most suitable subjects for this approach. In the standard approach to image restoration, an estimate of the original image is given by maximizing $a$ posteriori probability distribution (the MAP estimate) [1]. In the context of statistical mechanics, this approach corresponds to finding the ground-state configuration of the effective Hamiltonian for some spin system under random fields. On the other hand, it is possible to construct another strategy to infer the original image using the thermal equilibrium state of the Hamiltonian. From the Bayesian statistical point of view, the finite temperature restoration coincides with maximizing a posterior marginal distribution (the MPM estimate [2,3]) and by using this strategy the error for each pixel may become smaller than that of the MAP estimate. As we use the average of each pixel (spin) over the BoltzmannGibbs distribution at a specific temperature, thermal fluctuation should play an important role in the MPM estimate. Then the temperature controls the shape of the distribution and, if we choose the temperature appropriately, sampling from the distribution generates the important configurations for a good restoration. In addition to this hill-climbing mechanism by the thermal fluctuation, we may use another type of fluctuation, namely, the quantum fluctuation that leads to quantum tunneling between states. If we use sampling from the Boltzmann-Gibbs distribution based on quantum fluctuation, it may be possible to obtain much more effective configurations for a good restoration. The idea of the Markov random-field model using quantum fluctuation was recently proposed by Tanaka and Horiguchi [4]; however, they investigated the quantum fluctuation in the context of optimization (the MAP estimate by quantum fluctuation) and they used the ground state as the estimate of the original image. We would like to stress that we use a distribution based on the quantum fluctuation itself and the expectation value is used to infer the original image. It is a highly nontrivial problem to investigate whether the MPM estimate based on quantum fluctuation is better than the MAP estimate or the thermal fluctuation based MPM estimate. This is the essential concept of this paper.

This paper is organized as follows. In Sec. II, we explain our model system and the essential idea of our method in detail. We also introduce the criterion of the restoration, that is, the overlap between the original image and the result of the restoration. In Sec. III, we introduce the infinite range model in order to obtain analytical results for the performance of the restoration, and calculate the overlap explicitly. In Sec. IV, we show that quantum Monte Carlo simulations in two dimensions support our analytical results. In Sec. V, we introduce an iterative algorithm which is derived by the mean-field approximation and apply this algorithm to image restoration for standard pictures. Section VI is devoted to discussion of all results we obtain. In this section, we also mention the inequality that gives the upper bound of the overlap.

\section{ESSENTIAL IDEA AND FORMULATION}

Let us suppose that the original image is represented by a configuration of Ising spins $\{\xi\} \equiv\left\{\xi_{i} \mid \xi_{i}= \pm 1 ; i=1, \ldots, N\right\}$ with probability $P_{s}(\{\xi\})$. These images are sent through the noisy channel in the form of the sequence $\{\xi\}$. We regard the output of the sequence $\{\xi\}$ through the noisy channel as $\{\tau\}$. The output probability for the case of a binary symmetric channel (BSC) is specified by

$$
P_{\text {out }}(\{\tau\} \mid\{\xi\})=\frac{1}{\left(2 \cosh \beta_{\tau}\right)^{N}} \exp \left(\beta_{\tau} \sum_{i} \tau_{i} \xi_{i}\right)
$$

We understand the relevance of this expression for the BSC; let us suppose that each pixel $\xi_{i}$ changes its sign with probability $p_{\tau}$ and remains with $1-p_{\tau}$ during the transmission, that is,

$$
\begin{gathered}
P\left(\tau_{i}=-\xi_{i} \mid \xi_{i}\right)=p_{\tau} \equiv \frac{\mathrm{e}^{-\beta_{\tau}}}{2 \cosh \beta_{\tau}} \\
P\left(\tau_{i}=\xi_{i} \mid \xi_{i}\right)=1-p_{\tau} \equiv \frac{\mathrm{e}^{\beta_{\tau}}}{2 \cosh \beta_{\tau}} .
\end{gathered}
$$

We easily see that there is a simple relation between flip probability $p_{\tau}$ and inverse temperature $\beta_{\tau}$ as $\exp \left(2 \beta_{\tau}\right)$ $=\left(1-p_{\tau}\right) / p_{\tau}$. This is the reason why we refer to this type of noise as binary symmetric channel. Using the assumption that each pixel $\xi_{i}$ in the original image $\{\xi\}$ is corrupted independently (so-called memory-less channel) namely, 
$P(\{\tau\} \mid\{\xi\})=\Pi_{i} P\left(\tau_{i} \mid \xi_{i}\right)$, we obtain Eq. (1). This BSC is simply extended to the following Gaussian channel (GC)

$$
P_{\text {out }}(\{\tau\} \mid\{\xi\})=\frac{1}{(\sqrt{2 \pi} \tau)^{N}} \exp \left(-\frac{1}{2 \tau^{2}} \sum_{i}\left(\tau_{i}-\tau_{0} \xi_{i}\right)^{2}\right),
$$

where $\tau$ is a standard deviation of observable (corrupted pixel) $\tau_{i}$ from scaled original pixel $\tau_{0} \xi_{i}$.

Then the posterior probability $P(\{\sigma\} \mid\{\tau\})$, which is the probability that the source sequence is $\{\sigma\}$ provided that the output is $\{\tau\}$, leads to

$$
P(\{\sigma\} \mid\{\tau\})=\frac{P(\{\tau\} \mid\{\sigma\}) P_{m}(\{\sigma\})}{\sum_{\sigma} P(\{\tau\} \mid\{\sigma\}) P_{m}(\{\sigma\})}
$$

by the Bayes theorem. As we treat a black or white image and the BSC (1), a likelihood $P(\{\tau\} \mid\{\xi\})$ is appropriately written by

$$
P(\{\tau\} \mid\{\xi\}) \sim \exp \left(h \sum_{i} \tau_{i} \sigma_{i}\right)
$$

$P_{m}(\{\sigma\})$ appearing in the Bayesian formula (5) is a model of the prior distribution $P_{s}(\{\xi\})$, and we usually use the type

$$
P_{m}(\{\sigma\}) \sim \exp \left(\beta_{m} \sum_{\langle i j\rangle} \sigma_{i} \sigma_{j}\right)
$$

where $\Sigma_{\langle i j\rangle}(\cdots)$ means the sum with respect to the nearestneighboring pixels and $\beta_{m}$ controls the smoothness of the picture according to our assumption. Substituting Eqs. (6) and (22) into Eq. (8), we obtain the posterior probability $P(\{\sigma\} \mid\{\tau\})$ explicitly;

$$
P(\{\sigma\} \mid\{\tau\})=\frac{\exp \left(\beta_{m} \sum_{\langle i j\rangle} \sigma_{i} \sigma_{j}+h \sum_{i} \tau_{i} \sigma_{i}\right)}{\sum_{\sigma} \exp \left(\beta_{m} \sum_{\langle i j\rangle} \sigma_{i} \sigma_{j}+h \sum_{i} \tau_{i} \sigma_{i}\right)} .
$$

In the framework of the MAP estimate, we regard a configuration $\{\sigma\}$ that maximizes the posterior probability $P(\{\sigma\} \mid\{\tau\})$ as an estimate of the original image $\{\xi\}$. Obviously, this estimate $\{\sigma\}$ corresponds to the ground state of the following effective Hamiltonian (the random-field Ising model):

$$
\mathcal{H}_{\mathrm{eff}}=-\beta_{m} \sum_{\langle i j\rangle} \sigma_{i} \sigma_{j}-h \sum_{i} \tau_{i} \sigma_{i}
$$

Therefore, in the limit of $\beta_{m} / h \rightarrow \infty$, we expect that the original image should be a completely black or completely white picture, whereas in the limit of $\beta_{m} / h \rightarrow 0$, we assume that the original image should be identical to the observable $\{\tau\}$ itself.
On the other hand, in the framework of the MPM estimate, we maximize the following posterior marginal probability

$$
P\left(\sigma_{i} \mid\{\tau\}\right)=\frac{\sum_{\sigma \neq \sigma_{i}} P(\{\tau\} \mid\{\sigma\}) P_{m}(\{\sigma\})}{\sum_{\sigma} P(\{\tau\} \mid\{\sigma\}) P_{m}(\{\sigma\})} .
$$

As we treat the case of black or white image, the estimate of the $i$ th pixel should be given by

$$
\begin{aligned}
\operatorname{sgn}\left[\sum_{\sigma_{i}= \pm 1} \sigma_{i} P\left(\sigma_{i} \mid\{\tau\}\right)\right] \\
=\operatorname{sgn}\left(\frac{\sum_{\sigma} \sigma_{i} P(\{\tau\} \mid\{\sigma\}) P_{m}(\{\sigma\})}{\sum_{\sigma} P(\{\tau\} \mid\{\sigma\}) P_{m}(\{\sigma\})}\right) \\
\equiv \operatorname{sgn}\left(\left\langle\sigma_{i}\right\rangle_{h, \beta_{m}}\right)
\end{aligned}
$$

where $\langle\cdots\rangle_{h, \beta_{m}}$ means the average over the posterior probability Eq. (8). Consequently, our problem is reduced to one of statistical mechanics that is described by the effective Hamiltonian Eq. (9). As the Hamiltonian Eq. (9) has many local minima due to the quenched disorder $\{\tau\}$, in general, it is quite difficult to obtain the thermal equilibrium state that contributes to good restoration without being trapped in a local minimum for a long time. In order to overcome this difficulty, we add the quantum transverse field [5]

$$
-\Gamma \sum_{i} \hat{\sigma}_{i}^{x} \equiv \hat{\mathcal{H}}_{1}
$$

to the effective Hamiltonian (9) as a quantum fluctuation. In this expression, $\hat{\sigma}_{i}^{x}$ means the $x$ component of the Pauli matrix and $\Gamma$ controls the width of the quantum fluctuation. The term $\Gamma \hat{\sigma}_{i}^{x}$ can be understood intuitively as the tunneling probability between the eigenstates of the operator $\hat{\sigma}_{i}^{z}(z$ component of the Pauli matrix) namely, $\left|\sigma_{i}^{z}= \pm 1\right\rangle$. The tunneling probability between the states $\left|\sigma_{i}^{z}= \pm 1\right\rangle$ leads to $\left|\left\langle\sigma_{i}^{z}=+1\left|\Gamma \hat{\sigma}_{i}^{x}\right| \sigma_{i}^{z}=-1\right\rangle\right|^{2}=\Gamma^{2}$. As a result, the term (12) generates a superposition of the states $\left|\sigma_{i}^{z}=+1\right\rangle$ (black) and $\left|\sigma_{i}^{z}=-1\right\rangle$ (white). Using this fuzzy representation for each pixel, we may construct an algorithm that is robust for the choice of the hyperparameters, especially for the edge parts of a given picture.

Our problem is now reduced to one of quantum statistical mechanics for the effective Hamiltonian

$$
\hat{\mathcal{H}}_{\mathrm{eff}}=-h \sum_{i} \tau_{i} \hat{\sigma}_{i}^{z}-\beta_{m} \sum_{\langle i j\rangle} \hat{\sigma}_{i}^{z} \hat{\sigma}_{j}^{z}-\Gamma \sum_{i} \hat{\sigma}_{i}^{x} \equiv \hat{\mathcal{H}}_{0}+\hat{\mathcal{H}}_{1},
$$


where we defined $\hat{\mathcal{H}}_{1} \equiv \hat{\mathcal{H}}_{\text {eff }}-\hat{\mathcal{H}}_{0}$. Our main goal is to calculate the local magnetization $\left\langle\hat{\sigma}_{i}^{z}\right\rangle_{h, \beta_{m}, \Gamma}$ of the system described by the above Hamiltonian, that is to say,

$$
\left\langle\hat{\sigma}_{i}^{z}\right\rangle_{h, \beta_{m}, \Gamma} \equiv \frac{\operatorname{Tr}_{\sigma} \hat{\sigma}_{i}^{z} \exp \left(-\hat{\mathcal{H}}_{\mathrm{eff}}\right)}{\operatorname{Tr}_{\sigma} \exp \left(-\hat{\mathcal{H}}_{\mathrm{eff}}\right)}
$$

and we regard the quantity $\operatorname{sgn}\left(\left\langle\hat{\sigma}_{i}^{z}\right\rangle_{h, \beta_{m}, \Gamma}\right)$ as an estimate of the original pixel $\xi_{i}$. Therefore, the averaged performance of our method is measured by the overlap $M\left(h, \beta_{m}, \Gamma\right)$

$$
\begin{aligned}
& M\left(h, \beta_{m}, \Gamma\right) \\
& \quad=\operatorname{Tr}_{\{\xi, \tau\}} P_{s}(\{\xi\}) P_{\text {out }}(\{\tau\} \mid\{\xi\}) \xi_{i} \operatorname{sgn}\left(\left\langle\hat{\sigma}_{i}^{z}\right\rangle_{h, \beta_{m}, \Gamma}\right) .
\end{aligned}
$$

Thus, our main interests are summarized as follows. (i) Is it possible for us to use the quantum fluctuation in place of the thermal one? (ii) Does there exist a specific choice of $\Gamma$ that gives optimal image restoration?

Before we calculate the overlap (15), we may add the parity check term, which was recently introduced by Nishimori and Wong [6], to the effective Hamiltonian (9). This parity check term is represented as $\beta_{J} \Sigma_{\langle i j\rangle} J_{i j} \hat{\sigma}_{i}^{z} \hat{\sigma}_{j}^{z}$, and we rewrite $\hat{\mathcal{H}}_{0}$ as

$$
\hat{\mathcal{H}}_{0}=-\beta_{J} \sum_{\langle i j\rangle} J_{i j} \hat{\sigma}_{i}^{z} \hat{\sigma}_{j}^{z}-\beta_{m} \sum_{\langle i j\rangle} \hat{\sigma}_{i}^{z} \hat{\sigma}_{j}^{z}-h \sum_{i} \tau_{i} \hat{\sigma}_{i}^{z},
$$

where $J_{i j}$ is the noisy version of the product of two arbitrary original pixels $\xi_{i} \xi_{j}$ and the output of this quantity through the noisy channel is given by

$$
P_{\text {out }}(\{J\} \mid\{\xi\})=\frac{1}{\left(2 \cosh \beta_{r}\right)^{N_{B}}} \exp \left(\beta_{r} \sum_{\langle i j\rangle} J_{i j} \xi_{i} \xi_{j}\right)
$$

for the BSC and

$$
P_{\text {out }}(\{J\} \mid\{\xi\})=\frac{1}{(\sqrt{2 \pi} J)^{N_{B}}} \exp \left(-\frac{1}{2 J^{2}} \sum_{\langle i j\rangle}\left(J_{i j}-J_{0} \xi_{i} \xi_{j}\right)^{2}\right)
$$

for the GC, respectively. $N_{B}$ is the number of the terms appearing in the sum in Eq. (17) or Eq. (18). Thus the effective Hamiltonian $\hat{\mathcal{H}}_{\text {eff }}=\hat{\mathcal{H}}_{0}+\hat{\mathcal{H}}_{1}$ describes the thermodynamics of a quantum spin glass $[5,7]$ under random fields.

In the next section, we introduce a rather artificial model, namely, the infinite range model, in which spins in the system (13) are fully connected.

\section{THE INFINITE RANGE MODEL}

In this section, we calculate the overlap (15) explicitly using the infinite range version of the effective Hamiltonian (13). We use the GC for the analysis of the infinite range model in this section and the BSC for the quantum Monte Carlo simulations in Sec. IV. However, these two channels can be treated with the single form

$$
\begin{aligned}
P_{\text {out }}(\{J\} \mid\{\tau\})= & \prod_{\langle i j\rangle} F_{r}\left(J_{i j}\right) \prod_{\langle i j\rangle} F_{1}\left(\tau_{i j}\right) \\
& \times \exp \left(\beta_{r} \sum_{\langle i j\rangle} J_{i j} \xi_{i} \xi_{j}+\beta_{\tau} \sum_{i} \tau_{i} \xi_{i}\right)
\end{aligned}
$$

with

$$
\begin{gathered}
F_{r}\left(J_{i j}\right)=\frac{1}{2 \cosh \beta_{r}}\left\{\delta\left(J_{i j}-1\right)+\delta\left(J_{i j}+1\right)\right\}, \\
F_{1}\left(\tau_{i j}\right)=\frac{1}{2 \cosh \beta_{\tau}}\left\{\delta\left(\tau_{i}-1\right)+\delta\left(\tau_{i}+1\right)\right\}
\end{gathered}
$$

for the BSC and

$$
\begin{aligned}
& F_{r}\left(J_{i j}\right)=\frac{1}{\sqrt{2 \pi J^{2}}} \exp \left(-\frac{1}{2 J^{2}}\left(J_{i j}^{2}+J_{0}^{2}\right)\right), \\
& F_{1}\left(\tau_{i}\right)=\frac{1}{\sqrt{2 \pi \tau^{2}}} \exp \left(-\frac{1}{2 \tau^{2}}\left(\tau_{i}^{2}+\tau_{0}^{2}\right)\right),
\end{aligned}
$$

for the GC, and we set $\beta_{J}=J_{0} / J^{2}$ and $\beta_{\tau}=\tau_{0} / \tau^{2}$.

As the original image, we use the ferromagnetic snapshot from the distribution

$$
P_{s}(\{\xi\})=\frac{1}{\mathcal{Z}\left(\beta_{s}\right)} \exp \left(\frac{\beta_{s}}{N} \sum_{i j} \xi_{i} \xi_{j}\right)
$$

where $\Sigma_{i j}(\cdots)$ means the sum over all possible combinations of $(i, j)$ and we divided the argument of the exponential in Eq. (22) by $N$ to take a proper thermodynamic limit as the Hamiltonian should be of order $N$. For the same reason, we should rescale the terms appearing in Eq. (13) as $\beta_{J} \Sigma_{\langle i j\rangle} J_{i j} \hat{\sigma}_{i}^{z} \hat{\sigma}_{j}^{z} \rightarrow\left(\beta_{J} / N\right) \Sigma_{i j} J_{i j} \hat{\sigma}_{i}^{z} \hat{\sigma}_{j}^{z}$ and $\beta_{m} \Sigma_{\langle i j\rangle} \hat{\sigma}_{i}^{z} \hat{\sigma}_{j}^{z}$ $\rightarrow\left(\beta_{m} / N\right) \sum_{i j} \hat{\sigma}_{i}^{z} \hat{\sigma}_{j}^{z}$ when we treat the infinite range model.

It must be noted that $\hat{\mathcal{H}}_{0}$ and $\hat{\mathcal{H}}_{1}$ do not commute with each other and we use the Trotter decomposition [8]

$$
\mathcal{Z}=\lim _{P \rightarrow \infty} \operatorname{Tr}_{\sigma^{z}}\left(\mathrm{e}^{-\beta \mathcal{H}_{0} / P} \mathrm{e}^{-\beta \mathcal{H}_{1} / P}\right)^{P}
$$

to calculate the partition function explicitly. In this formula, $\mathcal{H}_{0}$ and $\mathcal{H}_{1}$ are eigenvalues of the operators $\hat{\mathcal{H}}_{0}$ and $\hat{\mathcal{H}}_{1}$ with respect to the eigenvector

$$
\left|\left\{\sigma_{k}^{z}\right\}\right\rangle=\prod_{i=1}^{N} \otimes\left|\sigma_{i k}^{z}\right\rangle \quad(k=1, \ldots, P)
$$

with

$$
\hat{\sigma}_{i k}^{z}\left|\sigma_{i k}^{z}\right\rangle \equiv \sigma_{i k}\left|\sigma_{i k}^{z}\right\rangle .
$$

$P$ means the Trotter number and we distinguish the different Trotter slices by the indices $k$. 
Now we can calculate the partition function for the quantum spin system (16) in terms of the corresponding classical spin system whose dimension increases by 1 . Using the Trotter formula (the path-integral formula) and well-known replica method [9], namely,

$$
\begin{gathered}
{[\ln \mathcal{Z}]=\lim _{n \rightarrow \infty} \frac{\left[\mathcal{Z}^{n}\right]-1}{n},} \\
{\left[\xi_{i}\right]=m_{0}=\tanh \left(\beta_{0} m_{0}\right),} \\
{\left[\left\langle\sigma_{i K}^{\alpha}\right\rangle_{\left.h, \beta_{m}, \Gamma\right]}=m=\frac{\operatorname{Tr}_{\xi} \mathrm{e}^{\beta_{s} m_{0} \xi}}{2 \cosh \left(\beta_{s} m_{0}\right)} \int_{-\infty}^{\infty} D u \Omega^{-1} \int_{-\infty}^{\infty} D \omega \Phi y^{-1} \sinh y,\right.} \\
{\left[\xi_{i}\left\langle\sigma_{i K}^{\alpha}\right\rangle_{\left.h, \beta_{m}, \Gamma\right]}=t\right.} \\
=\frac{\operatorname{Tr}_{\xi} \mathrm{e}^{\beta_{s} m_{0} \xi}}{2 \cosh \left(\beta_{s} m_{0}\right)} \int_{-\infty}^{\infty} D u \Omega^{-1} \int_{-\infty}^{\infty} D \omega \xi \Phi y^{-1} \sinh y ; \\
{\left[\left\langle\left(\sigma_{i K}^{\alpha}\right)^{2}\right\rangle_{\left.h, \beta_{m}, \Gamma\right]}=Q=\frac{\operatorname{Tr}_{\xi} \mathrm{e}^{\beta_{s} m_{0} \xi}}{2 \cosh \left(\beta_{s} m_{0}\right)} \int_{-\infty}^{\infty} D u\left[\Omega^{-1} \int_{-\infty}^{\infty} D \omega \Phi y^{-1} \sinh y\right]^{2},\right.} \\
{\left[\left\langle\sigma_{i K}^{\alpha} \sigma_{i L}^{\alpha}\right\rangle_{\left.h, \beta_{m}, \Gamma\right]}\right]=\frac{\operatorname{Tr}_{\xi} \mathrm{e}^{\beta_{s} m_{0} \xi}}{2 \cosh \left(\beta_{s} m_{0}\right)} \int_{-\infty}^{\infty} D u \Omega^{-1}\left[\int_{-\infty}^{\infty} D \omega \Phi^{2} y^{-2} \cosh y+\Gamma^{2} \int_{-\infty}^{\infty} D \omega y^{-3} \sinh y\right],}
\end{gathered}
$$

we can obtain the overlap as a function of the macroscopic parameters $\beta_{m}$ and $\Gamma$ by making use of the saddle-point method. The bracket $[\cdots]$ denotes the average over the distribution $P_{s}(\{\xi\}) P_{\text {out }}(\{J\},\{\tau\} \mid\{\xi\})$.

The standard replica calculations and saddle-point method where $\langle\cdots\rangle_{h, \beta_{m}, \Gamma}$ means the average by the posterior probability using the same method as Eq. (14). $D u$ or $D y$ means Gaussian integral measure $D u \equiv d u \mathrm{e}^{-u^{2} / 2} / \sqrt{2 \pi}$. In order to obtain the above saddle-point equations, we used the replica symmetric and the static approximations, that is,

$$
\begin{gathered}
t_{K}=t, \\
S_{\alpha}(K L)=S(K \neq L), \quad 1(K=L), \\
Q_{\alpha \beta}=Q .
\end{gathered}
$$

We also defined the functions $\Phi, y$, and $\Omega$ as

$$
\begin{gathered}
\Phi \equiv u \sqrt{(\tau h)^{2}+Q\left(J \beta_{J}\right)^{2}}+J \beta \omega \sqrt{S-Q}+\left(\tau_{0} h+J_{0} \beta_{J} t\right) \xi \\
+\beta_{m} m, \\
y \equiv \sqrt{\Phi^{2}+\Gamma^{2}} \\
\Omega \equiv \int_{-\infty}^{\infty} D \omega \cosh y
\end{gathered}
$$

Then the overlap, which is a measure of retrieval quality, is calculated explicitly as

$$
\begin{aligned}
{\left[\xi_{i} \operatorname{sgn}\left(\left\langle\sigma_{i K}^{\alpha}\right\rangle_{h, \beta_{d}, \Gamma}\right)\right]=} & M=\frac{\operatorname{Tr}_{\xi} \xi \mathrm{e}^{\beta_{s} m_{0} \xi}}{2 \cosh \left(\beta_{s} m_{0}\right)} \int_{-\infty}^{\infty} D u \int_{-\infty}^{\infty} D w \\
& \times \operatorname{sgn}\left[u \sqrt{(\tau h)^{2}+Q\left(J \beta_{J}\right)^{2}}\right. \\
& +\left(\tau_{0} h+J_{0} \beta_{J} t\right) \xi+\beta_{m} m \\
& \left.+J \beta_{J} w \sqrt{S-Q}\right]
\end{aligned}
$$

where the above overlap $M$ depends on $\Gamma$ through $m$ [Eq. (28)].

We first consider the case of $\beta_{J}=0$, that is to say, the conventional image restoration. We choose a snapshot from the distribution (22) at source temperature $T_{s}=0.9$. According to Nishimori and Wong [6], we fix the ratio $h / \beta_{m}$ and adjust $\beta_{m}\left(=1 / T_{m}\right)$ as a parameter for simulated annealing [10] and control $\Gamma$ as a quantum fluctuation. If we set $\Gamma$ $=0$, the lines of $M\left(T_{m}, \Gamma=0\right)$ should be identical to the results obtained by the thermal MPM estimate [6]. On the other hand, if we choose $T_{m}=0$ and $\Gamma=0$, the resultant line $M\left(T_{m}=0, \Gamma\right)$ represents the performance of the quantum MAP estimate. We should draw attention to the fact that the quantum fluctuation vanishes at $\Gamma=0$. In practical applications of the quantum annealing [11] based on quantum Monte Carlo simulations, we should reduce $\Gamma$ from $\Gamma>0$ to $\Gamma=0$ during Monte Carlo updates. However, the resultant performance obtained here is calculated analytically pro 


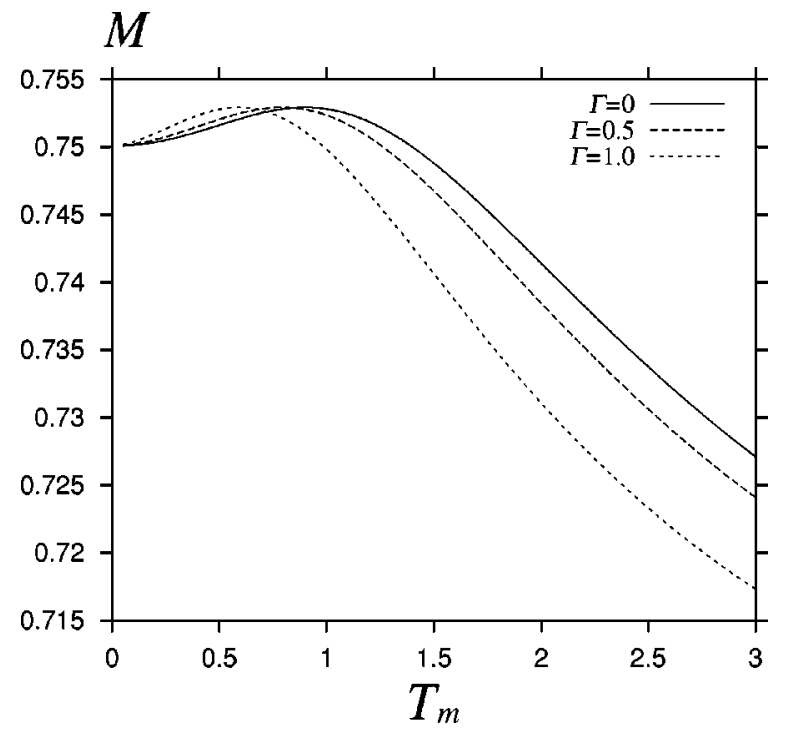

FIG. 1. The overlap $M$ as a function of $T_{m}$ for several values of $\Gamma$. We set the system parameters as $T_{s}=0.9, \tau_{0}=\tau=1.0$, and $h / \beta_{m}=0.9=\beta_{\tau} / \beta_{s}$. For $\Gamma=0$, the optimal temperature $T_{m}$ coincides with the source temperature $T_{s}=0.9$. As the quantum fluctuation $\Gamma$ increases, the optimal temperature is shifted to the lowtemperature region. However, the maximum value of the overlap does not change.

vided that the system reaches its equilibrium state. Therefore, we can regard the result $M\left(T_{m}=0, \Gamma=0\right)$ as a performance when $\Gamma$ is decreased slowly enough.

In Fig. 1, we set the ratio $h / \beta_{m}$ to its optimal value $\beta_{\tau} / \beta_{s}=0.9$ and plot the overlap $M\left(T_{m}, \Gamma\right)$ for the cases of $\Gamma=0,0.5$, and 1.0. Obviously, for the case of $\Gamma=0$, the maximum is obtained at a specific temperature $T_{m}=0.9$ $\left(=T_{s}\right)[6]$. However, if we add a finite quantum fluctuation, the optimal temperature $T_{m}$ is shifted to the low-temperature region.

In Fig. 2, we plot $M\left(T_{m}, \Gamma\right)$ for the cases of $T_{m}$ $=0,0.1,0.9$ with the fixed optimal ratio $h / \beta_{m}=0.9$. This figure shows that if we set the parameters $h, \beta_{m}$ to their optimal value in the thermal MPM estimate, the quantum fluctuation added to the system destroys the recovered image [see the lines $M\left(T_{m}, \Gamma\right)$ for the case of $\left.T_{m}=0.9\right]$. Therefore, we may say that it is impossible to choose all parameters $h, \beta_{m}$, and $\Gamma$ so as to obtain an overlap that is larger than $M_{\max }$ $\equiv M\left(T_{m}=0.9, \Gamma=0\right)$. This fact is also shown by a threedimensional plot $M\left(T_{m}, \Gamma\right)$ in Fig. 3.

Although, we found that a finite $\Gamma$ does not give the absolute maximum of the overlap, the quantum MPM estimate $M\left(T_{m}=0, \Gamma>0\right)$ has another kind of advantage. As Fig. 3 indicates, the overlap of the quantum MPM estimate is almost flat in comparison with $M\left(T_{m}=0.1, \Gamma>0\right)$ or $M\left(T_{m}\right.$ $=0.9, \Gamma>0)$. This is a desirable property from the practical point of view. This is because the estimation of the hyperparameters is one of the crucial problems in inferring the original image, and in general it is difficult to estimate them beforehand. Therefore, this robustness for hyperparameter selection is a desirable property. We also see this property in Fig. 3.

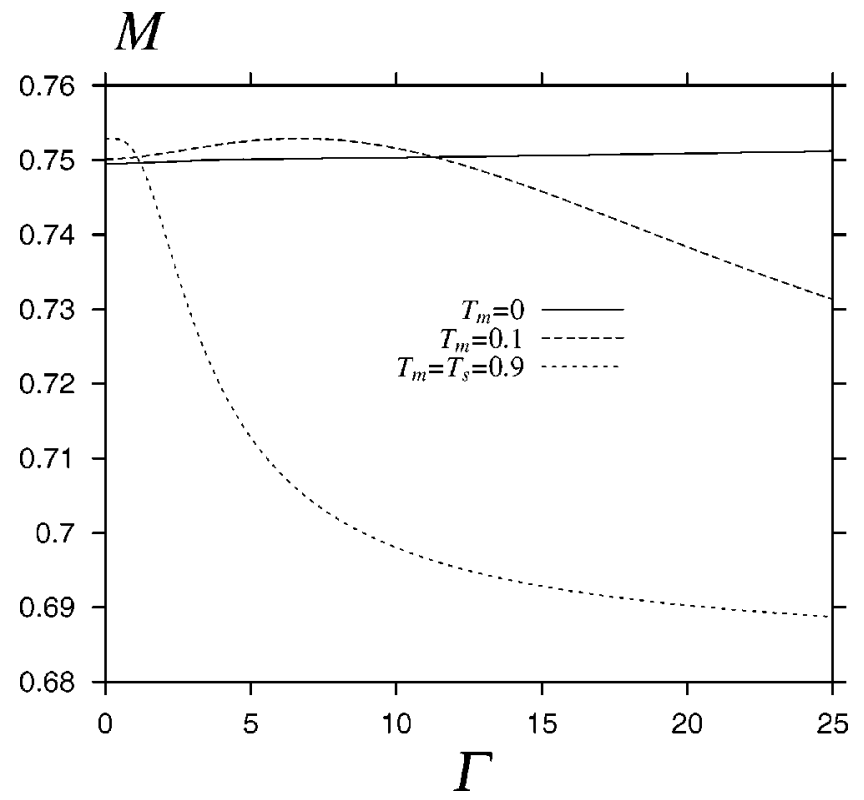

FIG. 2. The overlap $M$ as a function of $\Gamma$ for several values of $T_{m}$. We set the system parameters as $T_{s}=0.9, \tau_{0}=\tau=1.0$, and $h / \beta_{m}=0.9=\beta_{\tau} / \beta_{s}$. The overlap at $T_{m}=0$ and $\Gamma=0$ corresponds to the result obtained by quantum annealing. The quantum MPM estimate works effectively in the low-temperature region and the results are robust for the choice of $\Gamma$.

As we already mentioned, the overlap at $T_{m}=0$ and $\Gamma=0$ corresponds to the result that is obtained by quantum annealing [11], that is to say, the quantum MAP estimate. We see that the result of the quantum MPM estimate is slightly better than that of the quantum MAP estimate.

We next show the effect of the parity check term. In Fig. 4 , we set $T_{m}=T_{s}=0.9, h=1.0$, and $J_{0}=J=1.0$ and plot the overlap as a function of $\beta_{J}$ for several values of $\Gamma$. We see that the performance of the restoration is improved by introducing the parity check term, which has much information about the local structure of the original image.

In the next section, we check the usefulness of this method in terms of quantum Monte Carlo simulation.

\section{QUANTUM MONTE CARLO SIMULATION}

In this section, Monte Carlo simulations in realistic two dimensions are carried out in order to check the practical usefulness of our method. We use the standard pictures that are provided on the web site [12] as the original image, instead of the Ising snapshots. In order to sample the important points that contribute to the local magnetization $\left\langle\hat{\sigma}_{i}^{z}\right\rangle$, we use the quantum Monte Carlo method that was proposed by $\mathrm{Su}-$ zuki [8]. As we mentioned in the previous sections, we can treat the $d$-dimensional quantum system as a $(d+1)$ dimensional classical system by the Trotter decomposition [8]. In this sense, the transition probability of the Metropolis algorithm leads to 


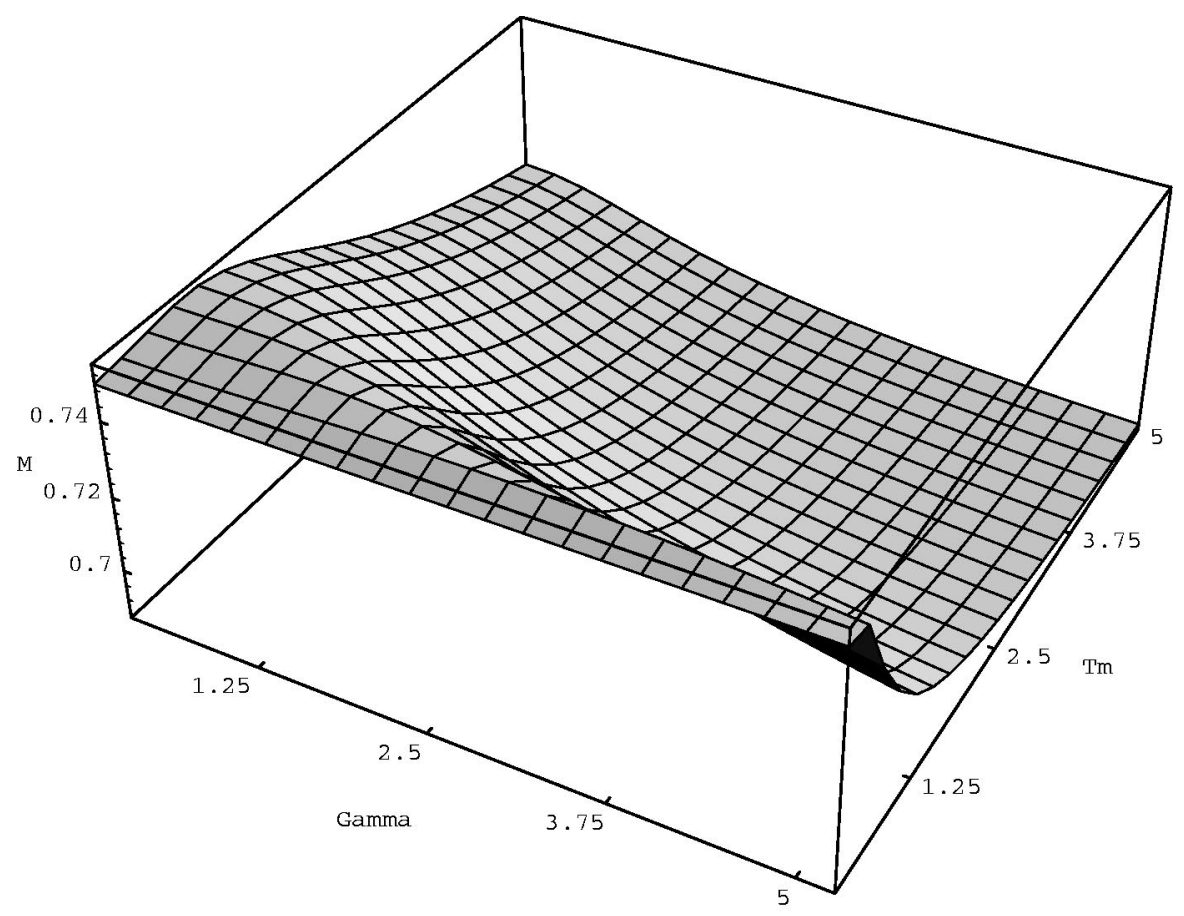

FIG. 3. The overlap $M$ as a function of the quantum fluctuation $\Gamma$ and temperature $T_{m}$.

$$
P\left(\{\sigma\} \rightarrow\{\sigma\}^{\prime}\right)=\min \left(1, \exp \left\{-\left[E\left(\{\sigma\}^{\prime}\right)-E(\{\sigma\})\right]\right\}\right)
$$

$E(\{\sigma\})$ is the energy of the classical spin system in $(d+1)$ dimensions [in the present case, $(2+1)=3$ dimensions $]$ and is given by

$$
\begin{aligned}
E(\{\sigma\}) \equiv & -\frac{\beta_{m}}{P} \sum_{i j k}\left[\sigma_{i, j, k} \sigma_{i+1, j, k}+\sigma_{i, j, k} \sigma_{i-1, j, k}\right. \\
& \left.+\sigma_{i, j, k} \sigma_{i, j+1, k}+\sigma_{i, j, k} \sigma_{i, j-1, k}\right]-\frac{h}{P} \sum_{i j k} \tau_{i, j} \sigma_{i, j, k} \\
& -B \sum_{i j k} \sigma_{i, j, k} \sigma_{i, j, k+1}
\end{aligned}
$$

where we defined $B \equiv \ln \cosh (\Gamma / P)$. The transition probability Eq. (39) with Eq. (40) generates the Boltzmann-Gibbs distribution asymptotically and, using the importance sampling from the distribution, we can calculate the expectation value of the $i$ th spin $\hat{\sigma}_{i}^{z}$, namely, $\left\langle\hat{\sigma}_{i}^{z}\right\rangle_{h, \beta_{m}, \Gamma}$. Using this result, we obtain an estimate of the $i$ th pixel of the original image as $\operatorname{sgn}\left(\left\langle\hat{\sigma}_{i}^{z}\right\rangle_{h, \beta_{m}, \Gamma}\right)$. We give the results in Figs. 5 and 6 . From these figures, we see that there exists an optimal value of the transverse field $\Gamma$. In Figs. 7 and 8, we display the results of quantum Monte Carlo simulations when we add the parity check term for the parameter sets $\Gamma=2.0, h$ $=1.0$, and $\beta_{m}=0.5$. We see that the resultant pictures with the parity check term are almost perfect $\left[\right.$ see $\beta_{J}=1.0$ and $1.5]$.

\section{MEAN-FIELD ALGORITHM}

In the previous sections, we see that quantum fluctuation works effectively in image restoration problems in the sense that the quantum fluctuations suppress the error of the hyperparameter's estimation in the Markov random-field model. In addition, by making use of the quantum Monte Carlo simulations, we could apply the method to the image restoration of two-dimensional standard pictures. However, in carrying out the simulations, it takes quite a long time to obtain the average $\left\langle\hat{\sigma}_{i}^{z}\right\rangle_{h, \beta_{m}, \Gamma}$ and this is not suitable for practical situations.

In this section, in order to overcome this computational

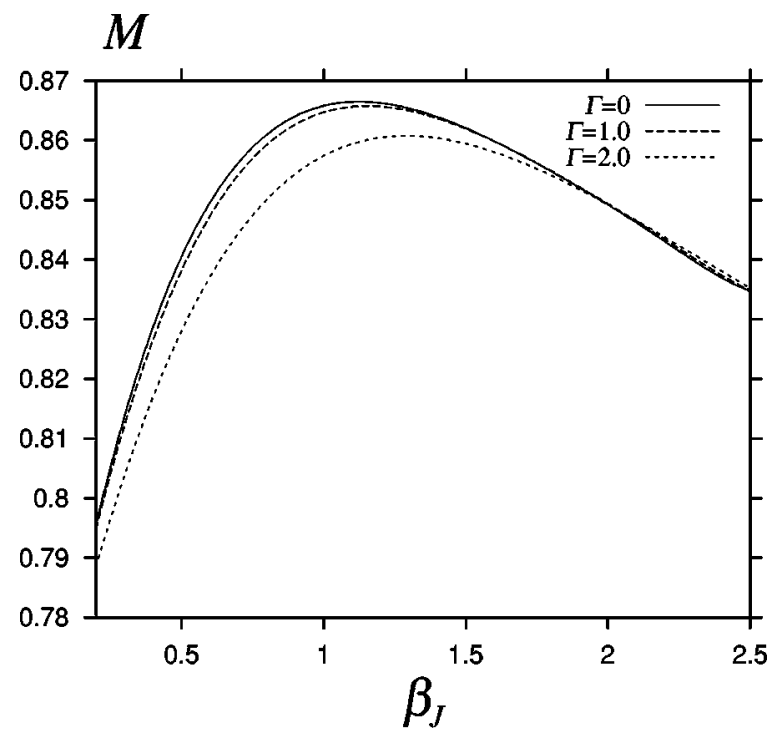

FIG. 4. The overlap $M$ as a function of $\beta_{J}$ for several values of $\Gamma$. We set the system parameters $T_{m}=T_{s}=0.9, \tau=\tau_{0}=1.0, J$ $=J_{0}=1.0$, and $h / \beta_{m}=0.9=\beta_{\tau} / \beta_{s}$. For the case of $\Gamma=0$, the optimal $\beta_{J}$ is naturally identical to $J_{0} / J^{2}=1.0$. As the quantum fluctuation $\Gamma$ increases, the overlap $M$ decreases because the quantum fluctuation destroys the recovered image. 

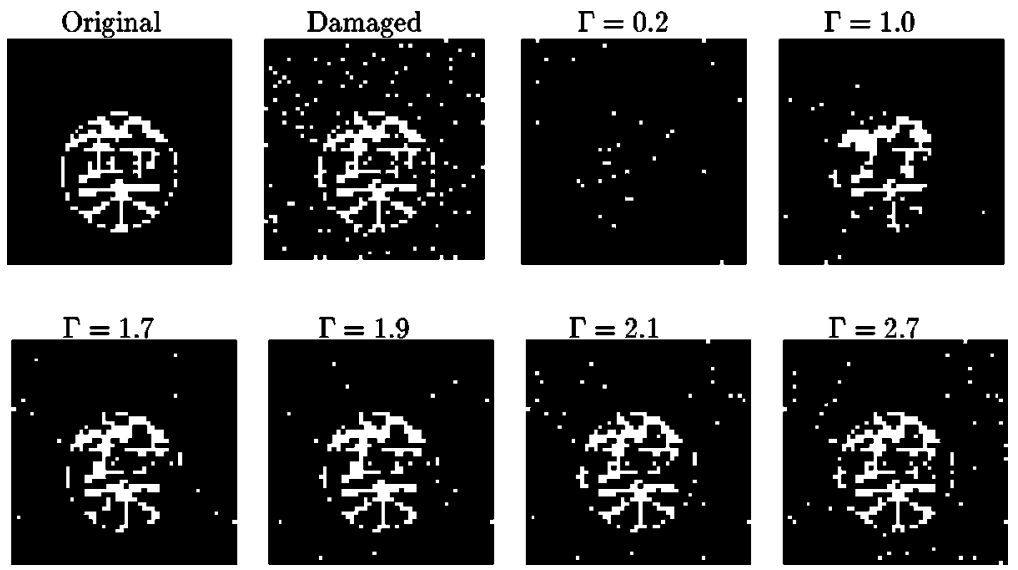

FIG. 5. The results by quantum Monte Carlo simulations for the standard picture (a Japanese kanji stamp for the name of "Suzuki," which is the most popular name in Japan; the size is $50 \times 50)$. From the upper left to the lower right, the original picture, the damaged picture, and the results for $\Gamma=0.2,1.0,1.7,1.9,2.1$, and 2.7 are displayed. The noise rate is $10 \%$. (The overlap between the original picture and the damaged one is 0.9.)

$$
\mathcal{Z}_{i j}=\mathrm{e}^{-\lambda_{i j}(1)}+\mathrm{e}^{-\lambda_{i j}(2)}
$$

the mean-field approximation. This algorithm shows fast convergence to the approximate solution. Within the meanfield approximation, we rewrite the density matrix $\hat{\rho}$ $=\mathrm{e}^{-\hat{\mathcal{H}}_{\text {eff }} / \mathcal{Z}}$ for the two-dimensional version of the effective Hamiltonian $\hat{\mathcal{H}}_{\text {eff }}$ as

$$
\hat{\rho} \simeq \prod_{i j} \otimes \hat{\rho}_{i j}
$$

where we defined $\hat{\rho}_{i j}$ as

$$
\hat{\rho}_{i j}=\sum_{n=1}^{2}\left|\sigma_{i j}(n)\right\rangle \mathrm{e}^{-\lambda_{i j}(n)}\left\langle\sigma_{i j}(n)\right|
$$

with

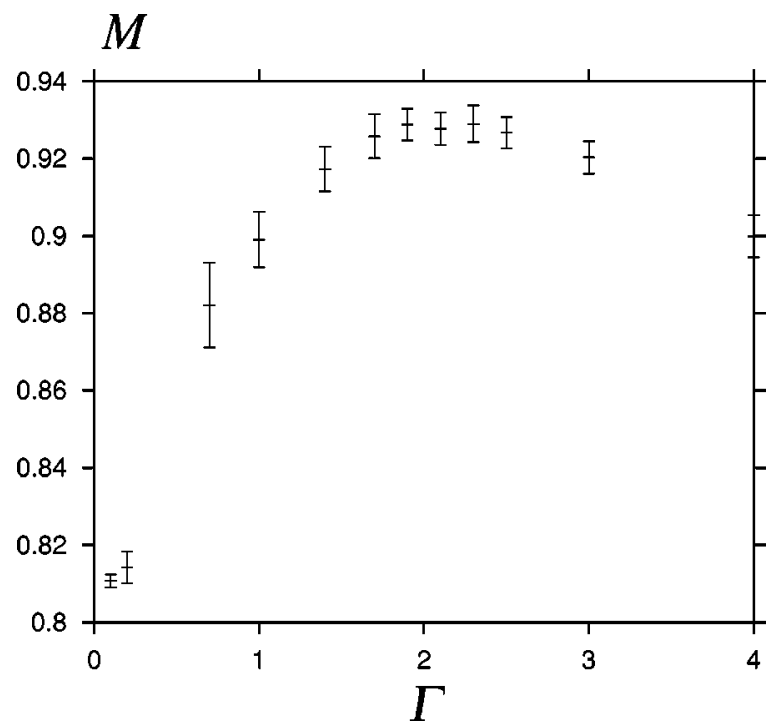

FIG. 6. The overlap $M$ as a function of the quantum fluctuation $\Gamma$ for the standard picture in Fig. 5. We set $\beta_{m}=0.5, h=1.0$, and $P=50$. The errorbars are calculated by averaging over five independent runs.
In the above expressions, $\lambda_{i j}(n), n=1,2$ means eigenvalues of the $2 \times 2$ matrix $\hat{\boldsymbol{H}}_{i j} \quad\left(\left[\hat{\boldsymbol{H}}_{i j}\right]_{11}=H_{i j}^{(+)},\left[\hat{\boldsymbol{H}}_{i j}\right]_{22}\right.$ $\left.=H_{i j}^{(-)}, \quad\left[\hat{\boldsymbol{H}}_{i j}\right]_{12}=\left[\hat{\boldsymbol{H}}_{i j}\right]_{21}=-\Gamma\right)$, and $H_{i j}^{( \pm)}$is defined by

$$
\begin{aligned}
H_{i j}^{(+)} & =-\left(\tau_{i j}+J m_{i+1, j}^{(t)}+J m_{i-1, j}^{(t)}+J m_{i, j+1}^{(t)}+J m_{i, j-1}^{(t)}\right) \\
& =-H_{i j}^{(-)} \equiv \alpha
\end{aligned}
$$

$$
J \equiv \frac{\beta_{m}}{h}=0.5
$$
at a site $(i, j)$, namely, $m_{i j}^{(t+1)}$, becomes
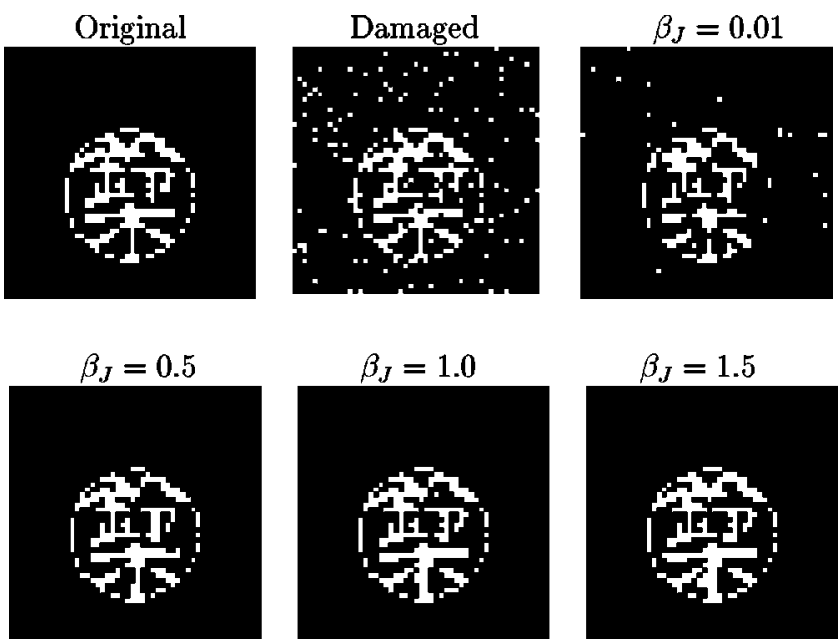

FIG. 7. The results by quantum Monte Carlo simulations including the parity check term. We fixed $\Gamma=2.0, h=1.0$, and $\beta_{m}=0.5$. From the upper left to the lower right, the original picture, the damaged picture, and the results of $\beta_{J}=0.01,0.5,1.0$, and 1.5 are shown. The noise rate is $10 \%$. (The overlap between the original picture and the damaged one is 0.9.)
Using this decoupled density matrix, the local magnetization 


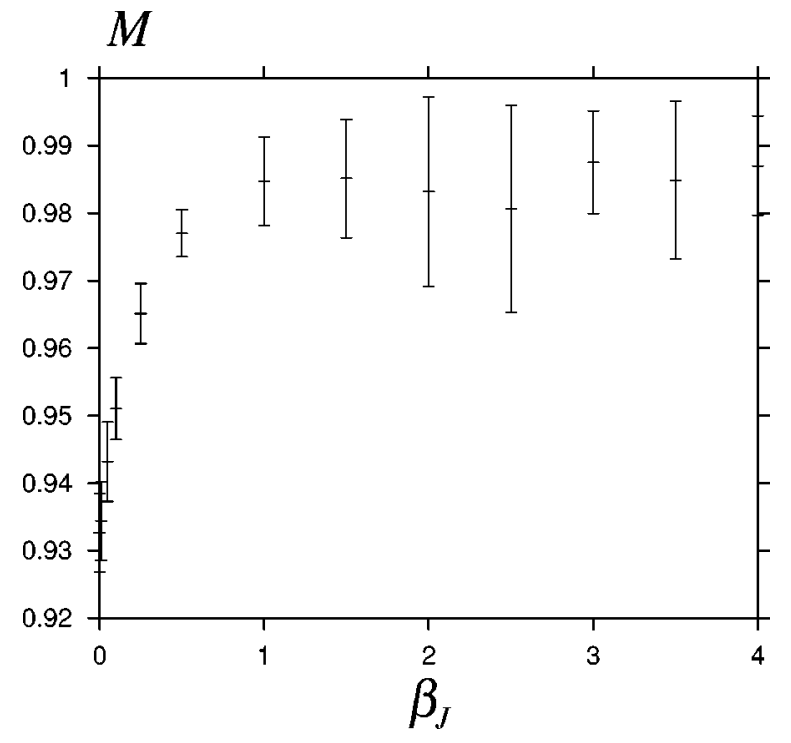

FIG. 8. $\beta_{J}$ dependence of the overlap $M$ calculated by quantum Monte Carlo simulations for the standard picture in Fig. 6. We set $\beta_{m}=0.5, h=1.0$, and $\Gamma=2.0$. The errorbars are calculated from five independent runs.

$$
\begin{aligned}
m_{i j}^{(t+1)}= & \operatorname{Tr}\left[\sigma_{i j}^{z} \hat{\rho}_{i j}\right] \\
= & \frac{\mathrm{e}^{\sqrt{\alpha^{2}+\Gamma^{2}}}}{2 \cosh \left(\sqrt{\alpha^{2}+\Gamma^{2}}\right)}\left[\frac{\left(\alpha+\sqrt{\alpha^{2}+\Gamma^{2}}\right)^{2}-\Gamma^{2}}{\left(\alpha+\sqrt{\alpha^{2}+\Gamma^{2}}\right)^{2}+\Gamma^{2}}\right] \\
& +\frac{\mathrm{e}^{-\sqrt{\alpha^{2}+\Gamma^{2}}}}{2 \cosh \left(\sqrt{\alpha^{2}+\Gamma^{2}}\right)}\left[\frac{\left(\alpha-\sqrt{\alpha^{2}+\Gamma^{2}}\right)^{2}-\Gamma^{2}}{\left(\alpha-\sqrt{\alpha^{2}+\Gamma^{2}}\right)^{2}+\Gamma^{2}}\right] .
\end{aligned}
$$

For this local magnetization (46), the estimate of the pixel $\xi_{i j}$ is obtained as $\operatorname{sgn}\left[m_{i j}\right]$.

We solve the mean-field equations (46) with respect to $m_{i j}$ until the condition

$$
\varepsilon_{i j} \equiv\left|m_{i j}^{(t+1)}-m_{i j}^{(t)}\right|<10^{-5}
$$

holds for all pixels $\{i, j\}$. We show its performance in Fig. 9 and Table I. From Table I, we see that, if we introduce
TABLE I. The overlap $M$ calculated by the quantum iterative algorithm for several values of $\Gamma$. The restored pictures are shown in Fig. 9. The iteration times are also listed. We set $J=0.5$.

\begin{tabular}{ccc}
\hline \hline$\Gamma$ & $M$ & Iteration times \\
\hline 0.001000 & 0.919200 & 21 \\
0.800000 & 0.921600 & 22 \\
1.200000 & 0.927200 & 8 \\
1.600000 & 0.932800 & 4 \\
2.400000 & 0.900800 & 2 \\
3.000000 & 0.840000 & 4 \\
\hline \hline
\end{tabular}

the appropriate quantum fluctuation, the performance is remarkably improved, and in addition, the speed of the convergence becomes much faster. However, if we add too much quantum fluctuation, the fluctuation destroys the recovered image. We also see that an optimal value of $\Gamma$ exists around $\Gamma \sim 1.6$.

\section{SUMMARY AND DISCUSSION}

In this paper, we investigated to what extent quantum fluctuation works effectively on image restoration. For this purpose, we introduced an analytically solvable model, that is, the infinite range version of the Markov random-field model. We applied the techniques of statistical mechanics to this model and derived the overlap explicitly. We found that the quantum fluctuation improves the quality of the image restoration dramatically in the low-temperature region. In this sense, the error of the estimation for the hyperparameters $\beta_{m}, h$ can be suppressed by the quantum fluctuation.

However, we also found that the maximum value of the overlap $M_{\max }^{\text {(quantum) }}$ never exceeds that of the classical Ising case $M_{\mathrm{max}}^{\text {(thermal) }}$. We may show this fact by the following arguments. First of all, the upper bound of the overlap for the classical system is given by setting $h=\beta_{\tau}$ and $P_{s}=P_{m}$, that is,

$$
\begin{aligned}
M_{\text {max }}^{\text {(thermal) }}\left(\beta_{\tau}, P_{s}\right) & =\operatorname{Tr}_{\{\tau, \xi\}} \xi_{i} \exp \left(\beta_{\tau} \sum_{i} \tau_{i} \xi_{i}\right) P(\{\xi\}) \operatorname{sgn}\left[\operatorname{Tr}_{\sigma} \sigma_{i} \exp \left(\beta_{\tau} \sum_{i} \tau_{i} \sigma_{i}\right) P_{m}(\{\sigma\})\right] \\
& =\operatorname{Tr}_{\{\tau, \xi\}} \xi_{i} \exp \left(\beta_{\tau} \sum_{i} \tau_{i} \xi_{i}\right) P(\{\xi\}) \frac{\operatorname{Tr}_{\sigma} \sigma_{i} \exp \left(\beta_{\tau} \sum_{i} \tau_{i} \sigma_{i}\right) P_{m}(\{\sigma\})}{\left|\operatorname{Tr}_{\sigma} \sigma_{i} \exp \left(\beta_{\tau} \sum_{i} \tau_{i} \sigma_{i}\right) P_{m}(\{\sigma\})\right|} \\
& =\operatorname{Tr}_{\tau}\left|\operatorname{Tr}_{\sigma} \sigma_{i} \exp \left(\beta_{\tau} \sum_{i} \tau_{i} \sigma_{i}\right) P_{m}(\{\sigma\})\right| .
\end{aligned}
$$

For the quantum system, the overlap is bounded by this maximum value $M_{\max }^{(\text {thermal })}$ as 

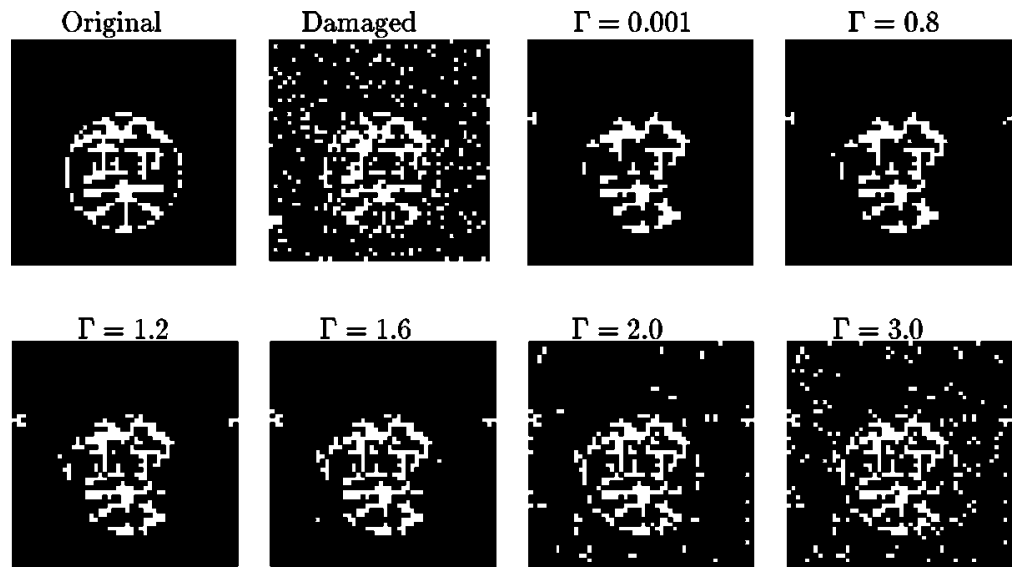

FIG. 9. The restored pictures (their size is $50 \times 50$ ) obtained with the quantum iterative algorithm for several values of $\Gamma$. From the upper left to the lower right, the original image, the corrupted image, the results of $\Gamma$ $=0.001,0.8,1 \cdot 2,1.6,2.0$, and $\Gamma=3.0$. The noise rate is $20 \%$. (The overlap between the original picture and the damaged one is 0.8.)

$$
\begin{aligned}
M^{\text {(quantum) }\left(h, P_{m}, \Gamma\right)} & =\operatorname{Tr}_{\{\tau, \xi\}} \xi_{i} \exp \left(\beta_{\tau} \sum_{i} \tau_{i} \xi_{i}\right) P(\{\xi\}) \operatorname{sgn}\left[\operatorname{Tr}_{\hat{\sigma}} \hat{\sigma}_{i}^{z} \exp \left(h \sum_{i} \tau_{i} \hat{\sigma}_{i}^{z}+\Gamma \sum_{i} \hat{\sigma}_{i}^{x}\right) P_{m}\left(\hat{\sigma}^{z}\right)\right] \\
& \leqslant\left|\operatorname{Tr}_{\{\tau, \xi\}} \xi_{i} \exp \left(\beta_{\tau} \sum_{i} \tau_{i} \xi_{i}\right) P(\{\xi\}) \operatorname{sgn}\left[\operatorname{Tr}_{\hat{\sigma}}^{\hat{\sigma_{i}}} \hat{\sigma}_{i}^{z} \exp \left(h \sum_{i} \tau_{i} \hat{\sigma}_{i}^{z}+\Gamma \sum_{i} \hat{\sigma}_{i}^{x}\right) P_{m}\left(\left\{\hat{\sigma}_{i}^{z}\right\}\right)\right]\right| \\
& =\operatorname{Tr}_{\tau}\left|\operatorname{Tr}_{\xi} \xi_{i} \exp \left(\beta_{\tau} \sum_{i} \tau_{i} \xi_{i}\right) P(\{\xi\})\right|=M_{\text {max }}^{\text {(thermal })} .
\end{aligned}
$$

We can see this inequality more directly as follows:

$$
\begin{aligned}
& \operatorname{Tr}_{\tau}\left|\operatorname{Tr}_{\xi} \xi_{i} \exp \left(\beta_{\tau} \sum_{i} \tau_{i} \xi_{i}\right) P(\{\xi\})\right| \geqslant \operatorname{Tr}_{\{\tau, \xi\}} \xi_{i} \exp \left(\beta_{\tau} \sum_{i} \tau_{i} \xi_{i}\right) P(\{\xi\}) \frac{\operatorname{Tr}_{\hat{\sigma}} \hat{\sigma}_{i}^{z} \exp \left(h \sum_{i} \tau_{i} \hat{\sigma}_{i}^{z}+\Gamma \sum_{i} \hat{\sigma}_{i}^{x}\right) P_{m}\left(\left\{\hat{\sigma}^{z}\right\}\right)}{\operatorname{Tr}_{\hat{\sigma}} \hat{\sigma}_{i}^{z} \exp \left(h \sum_{i} \tau_{i} \hat{\sigma}_{i}^{z}+\Gamma \sum_{i} \hat{\sigma}_{i}^{x}\right) P P_{m}\left(\left\{\hat{\sigma}^{z}\right\}\right) \mid} \\
&=\operatorname{Tr}_{\{\tau, \xi\}} \xi_{i} \exp \left(\beta_{\tau} \sum_{i} \tau_{i} \xi_{i}\right) P(\{\xi\}) \operatorname{sgn}\left[\operatorname{Tr}_{\hat{\sigma}} \hat{\sigma}_{i}^{z} \exp \left(h \sum_{i} \tau_{i} \hat{\sigma}_{i}^{z}+\Gamma \sum_{i} \hat{\sigma}_{i}^{x}\right) P_{m}\left(\left\{\hat{\sigma}^{z}\right\}\right)\right] \\
&=M^{\text {(quantum })}\left(h, P_{m}, \Gamma\right),
\end{aligned}
$$

where the identity $\operatorname{sgn}(x)=x /|x|$ was used. We should notice that in the left-hand side of the above inequality (50), the arguments of the trace with respect to $\tau$ always take positive values, while in the right-hand side they can be negative.

In order to check the usefulness of the method, we carried out quantum Monte Carlo simulations in realistic two dimensions. We found that the results of the simulation support qualitative behavior of the analytical expressions for overlap.

We introduced an iterative algorithm in terms of the mean-field approximation and applied it to image restoration of standard pictures. We found that the quantum fluctuations suppress the error in the hyperparameter estimation. In addition, we found that the speed of the convergence to the solution is accelerated by the quantum fluctuations.

From all the results obtained in this paper, we conclude that the quantum fluctuation turns out to enhance tolerance of uncertainties in hyperparameter estimation. However, if much higher quality of restoration is required, we must estimate those parameters using some method. One strategy for this purpose is selecting the parameters $\beta_{m}, h$, and $\Gamma$ that maximize a marginal likelihood. By making use of the infinite range model, the usefulness of this method can be evaluated. The details of the analysis will be reported in a forthcoming paper. Of course, the application of this strategy to the restoration of gray-scaled images $[13,14]$ will be considered as an important future problem.

\section{ACKNOWLEDGMENTS}

The author acknowledges H. Nishimori for fruitful discussions and useful comments. He also thanks K. Tanaka for a kind tutorial on the theory of image restoration and drawing his attention to Ref. [4]. He acknowledges D. Bolle', A. C. C. Coolen, D. M. Carlucci, T. Horiguchi, P. Sollich, and K. Y. M. Wong for valuable discussions. The author thanks the Department of Physics, Tokyo Institute of Technology and the Department of Mathematics, Kings College, University 
of London, for hospitality. This work was partially supported by the Ministry of Education, Science, Sports and Culture of Japan, Grants-in-Aid for Encouragement of Young Scien- tists, Grant Nos. 11740225, 1999-2000. This work was also supported by the collaboration program between the Royal Society and the Japanese Physical Society.
[1] S. Geman and D. Geman, IEEE Trans. Pattern Anal. Mach. Intell. 6, 721 (1984).

[2] J. Marroquin, S. Mitter, and T. Poggio, J. Am. Stat. Assoc. 82, 76 (1987).

[3] J.M. Pryce and A.D. Bruce, J. Phys. A 28, 511 (1995).

[4] K. Tanaka and T. Horiguchi, Trans. Inst. Electron. Inf. Commun. Eng., J80-A-12, 2117 (1997) (in Japanese).

[5] K. Chakrabarti, A. Dutta, and D. Sen, Quantum Ising Phases and Transitions in Transverse Ising Models, Lecture Note in Physics 41 (Springer-Verlag, Berlin; 1996).

[6] H. Nishimori and K.Y.M. Wong, Phys. Rev. E 60, 132 (1999).
[7] A.J. Bray and M.A. Moore, J. Phys. C 13, L655 (1985).

[8] M. Suzuki, Prog. Theor. Phys. 56, 1454 (1976).

[9] D. Sherrington and S. Kirkpatrick, Phys. Rev. Lett. 35, 1792 (1975).

[10] S. Kirkpatrick, C.D. Gelatt, Jr., and M.D. Vecchi, Science 220, 671 (1983).

[11] T. Kadowaki and H. Nishimori, Phys. Rev. E 58, 5355 (1998).

[12] The standard pictures that are used in this paper are available at ftp://ftp.lab1.kuis.kyoto-u.ac.jp/pub/sidba/

[13] D.M. Carlucci and J. Inoue, Phys. Rev. E 60, 2547 (1999).

[14] J. Inoue and D.M. Carlucci (unpublished). 